\title{
Análise do perfil epidemiológico das intoxicações exógenas por medicamentos no
}

\section{Piauí, 2007 a 2019}

Analysis of the epidemiological profile of exogenous drug intoxications in the state of Piauí - Brazil, from 2007 to 2019

Análisis del perfil epidemiológico de las intoxicaciones por drogas exógenas en Piauí, 2007 a 2019

Ana Klara Rodrigues Alves

ORCID: https://orcid.org/0000-0002-1216-9386 Universidade Estadual do Piauí, Brasil E-mail:klaraphb@outlook.com

Barbara Beatriz Lira da Silva

ORCID: https://orcid.org/0000-0001-9166-6147 Universidade Estadual do Piauí, Brasil

E-mail: brbeatriz16@gmail.com

Breno Carvalho de Almeida

ORCID: https://orcid.org/0000-0001-8736-9481

Universidade Estadual do Piauí, Brasil

E-mail:carvalhobreno019@gmail.com

Raquel de Brito Pereira

ORCID: https://orcid.org/0000-0002-4455-4449 Universidade Estadual do Piauí, Brasil

E-mail: raquelbritopp@gmail.com

Larissa dos Santos Silva

ORCID: https://orcid.org/0000-0001-8485-4094 Universidade Estadual do Piauí, Brasil

E-mail: 1ssilva@ aluno.uespi.br

Ana Kamila Rodrigues Alves

ORCID: https://orcid.org/0000-0001-8045-3672 Universidade Federal do Piauí, Brasil

E-mail: kah_rod@hotmail.com

Ana Karla Rodrigues Alves

ORCID: https://orcid.org/0000-0002-6841-5566

Universidade Federal do Piauí, Brasil

E-mail: annka_ra@hotmail.com

Annderson Carneiro de Oliveira

ORCID: https://orcid.org/0000-0002-7363-5141

Universidade Mauricio de Nassau, Brasil

E-mail: annderson.ac@gmail.com

Érika Maria Andrade Silva

ORCID: https://orcid.org/0000-0001-5506-6478

Universidade Estadual do Sudoeste da Bahia, Brasil

E-mail: erikamariaans@gmail.com

Fábio Dias Nogueira

ORCID: https://orcid.org/0000-0001-7303-1180

Faculdade de Ciências Humanas, Exatas e da Saúde do Piauí, Brasil

E-mail: fdnvaq@gmail.com

Ricardo Vieira Bezerra Luz Rodrigues

ORCID: https://orcid.org/0000-0003-1429-9966

Faculdade de Ciências Humanas, Exatas e da Saúde do Piauí, Brasil

E-mail: ricardovblrodrigues@hotmail.com

Francisco Robson de Oliveira Alves

ORCID: https://orcid.org/0000-0002-9854-6460 Universidade Federal do Piaú, Brasil

E-mail: robson_oliveira.fisio@outlook.com

Gustavo Wilson de Sousa Mello

ORCID: https://orcid.org/0000-0001-6000-3869 Universidade Estadual do Piauí, Brasil

E-mail: gustavowsm@ hotmail.com

Hoanna Izabely Rêgo Castro

ORCID: https://orcid.org/0000-0001-5646-4902 Universidade Federal do Piauí, Brasil

E-mail: hoannacastro@gmail.com 


\author{
Daniel Rodrigues de Farias \\ ORCID: https://orcid.org/0000-0002-9530-8053 \\ Universidade Estadual do Piauí, Brasil \\ E-mail: drfphb@hotmail.com
}

\begin{abstract}
Resumo
A extensa variedade de medicamentos, a falta de conhecimento das suas propriedades, fácil acesso e seu uso excessivo podem causar danos à saúde, inclusive óbitos, representando um desafio para os serviços de saúde. O presente trabalho teve como objetivo analisar de forma retrospectiva o perfil epidemiológico das notificações de intoxicações agudas causadas por medicamentos ocorridas no Piauí, no período de 2007 até 2019. Trata-se de um estudo epidemiológico, descritivo, do tipo quantitativo, analisando o perfil epidemiológico das intoxicações medicamentosas notificadas no Piauí, no período de 2007 a 2019. Foram notificados 6.210 casos durante o período analisado, revelando-se 2019 como o ano de maior número de intoxicações por medicações em valores absolutos $(17,74 \%)$. Com relação ao número de notificações na zona urbana $(n=5.189)$ em que o gênero feminino é predominante $(n=4.433)$, prevalecendo às faixas etárias de 20-39 anos $(n=2.510)$ e 15-19 anos $(n=1.012)$. A tentativa de suicídio foi responsável por 3.450 casos. O presente estudo visa contribuir para o aprimoramento das políticas públicas de saúde com foco na prevenção, orientação e adoção de medidas que minimizem este quadro, com atenção especial para público adulto-jovem e feminino que são os principais responsáveis pelas notificações de intoxicação medicamentosa no Piauí.
\end{abstract}

Palavras-chave: Intoxicação exógena; Epidemiologia; Medicamento.

\begin{abstract}
The estensive variety of drugs, the lack of knowledge of their properties and their overuse can cause damage to to the patient's health, including deaths, representing a challenge for health services. This study had as objective to retrospectively analyze the epidemiological profile of notifications of acute drug poisoning that occurred in Piauí, in the period from 2007 to 2019. This is an epidemiological, descriptive, quantitative study, analyzing the epidemiological profile of drug poisonings reported in Piauí, from 2007 to 2019. In Piauí, 6,210 cases were reported during the period analyzed, revealing 2019 was the year with the highest number of drug poisonings in absolute values $(17,74 \%)$. Regarding the number of notifications in the urban area $(n=5,189)$ where the female gender is predominant $(n=4,433)$, prevailing the age groups of 20-39 years $(n=2,510)$ and $15-19$ years $(n=1,012)$. The suicide attempt was responsible for 3,450 cases. This study aims to contribute to the improvement of public health policies with a focus on prevention, guidance and the adoption of measures to minimize this situation, with special attention to young adults and women who are the main responsible for notifications of drug poisoning in Piauí.
\end{abstract}

Keywords: Exogenous intoxication; Epidemiology; Medicines.

\title{
Resumen
}

La gran variedad de medicamentos, el desconocimiento de sus propiedades y su uso excesivo pueden causar daños a la salud, incluida la muerte, lo que representa un desafío para los servicios de salud. Este estudio tuvo como objetivo analizar retrospectivamente el perfil epidemiológico de las notificaciones de intoxicación aguda por drogas ocurridas en Piauí, de 2007 a 2019. Se trata de un estudio epidemiológico, descriptivo, cuantitativo, que analiza el perfil epidemiológico de las intoxicaciones por drogas notificadas en Piauí, de 2007 a 2019. En Piauí, se reportaron 6.210 casos durante el período analizado, revelando 2019 como el año con mayor número de intoxicaciones por drogas en valores absolutos $(17,74 \%)$. En cuanto al número de notificaciones en el área urbana $(\mathrm{n}=5.189)$ donde predomina el género femenino $(\mathrm{n}=4.433)$, predominan los grupos de edad de 20-39 años $(\mathrm{n}=2.510)$ y $15-19$ años $(\mathrm{n}=1.012)$. El intento de suicidio fue responsable de 3.450 casos. Este estudio tiene como objetivo contribuir a la mejora de las políticas de salud pública con un enfoque en la prevención, orientación y adopción de medidas para minimizar esta situación, con especial atención a los adultos jóvenes y mujeres que son los principales responsables de las notificaciones de intoxicación por drogas en Piauí.

Palabras clave: Intoxicación exógena; Epidemiología; Medicamento.

\section{Introdução}

A exposição a agentes potencialmente tóxicos é uma situação comum no meio que nos encontramos. O processo de intoxicação ocorre quando uma substância encontrada no ambiente (água, alimento, plantas) ou de forma isolada (pesticida, medicamento, produto de uso industrial ou doméstico) é ingerida e/ou entra em contato com a pele, olhos ou mucosas. Dessa forma, desencadeando efeitos nocivos, os quais se manifestam por sinais, sintomas e alterações no funcionamento do organismo. Assim podendo evoluir de forma negativa e em alguns casos levar ao óbito (Santos, Neto \& Cunha, 2015; Toscano, Landim, Rocha \& Muñoz ,2016). 
De acordo com Lopes et al. (2019) a intoxicação medicamentosa se apresenta como uma evolução de sinais e sintomas decorrentes da ação de uma ou mais substâncias ingeridas, inaladas, injetadas, ou que, entraram em contato com mucosas e pele, em quantidades acima da ação terapêutica, determinando um estado de desequilíbrio do organismo e promovendo alterações nas funções orgânicas.

O crescente uso de substâncias químicas, não acompanhadas de medidas de controle, configura um alto risco para a saúde das pessoas expostas. Os medicamentos são produtos com finalidade profilática, curativa e paliativa (Huynh et al., 2018). Contudo, a extensa variedade de medicamentos, a falta de conhecimento das suas propriedades e seu uso excessivo podem causar danos à saúde do paciente, inclusive óbitos, representando um desafio à saúde pública em países desenvolvidos e em desenvolvimento, como o Brasil (Gonçalves, Santos, Gonçalves, Sarturi \& Junior, 2017).

Em 2004, segundo a Organização Mundial da Saúde (OMS), cerca de 350 mil casos de mortes por intoxicações medicamentosas ocorrem no mundo, devendo haver atenção especial às crianças. Como as mesmas necessitam de doses menores de medicamentos, qualquer aumento dessa quantidade pode levar a alterações graves (Domingos, Borghesan, Merino \& Higarashi, 2016).

No Brasil, conforme informações do Sistema Nacional de Informações Tóxico-Farmacológicas (SINITOX) registraram-se em 2017 um total de 76.115 casos de intoxicações humanas. Os medicamentos apresentaram-se como principal agente tóxico, respondendo por aproximadamente, $27,11 \%$ dos casos de intoxicação e 50 óbitos. A Região Nordeste está na terceira posição no ranking geral de intoxicações no país, com um número de 9.222 casos notificados, atrás das regiões Sul $(n=39.437)$ e Sudeste $(n=24.669)$. O número de casos notificados por exposição a medicamentos no Nordeste é de 670, representando 3,25\% do total de 20.637 em todo o Brasil (SINITOX, 2017).

São parâmetros que contribuem significativamente para os elevados índices de intoxicações por medicamentos: a facilidade na aquisição de fármacos sem prescrição médica; a reduzida regulação da publicidade acerca do medicamento; uma variedade de fármacos com segurança e eficácia duvidosas, a carência de uma legislação específica sobre embalagens seguras; falha em iniciativas de desenvolvimento da atenção farmacêutica; a multiplicação de redes de farmácia e drogarias; maus hábitos e erros nas prescrições médicas e de dispensação farmacêutica (Nobrega, Costa \& Mariz, 2015). Também contribuem para as intoxicações o padrão do consumo de medicamentos pela população (caracterizado pela automedicação); o uso inapropriado e indiscriminado, principalmente de psicotrópicos e antibióticos; e a debilidade nas medidas preventivas, de fiscalização e controle por parte das autoridades (Almeida, 2015).

Várias são as circunstâncias que podem levar a um quadro de intoxicação: exposição profissional ou acidental (Mendonça et al., 2016; Parand, Garfield \& Vincent, 2016), abuso (Nguyen, Pizon, Branas \& Anthony, 2016), tentativa de suicídio (Geulayov et al., 2018) e homicídio (Moyo, Zhao \& Thorpe, 2019). Intoxicações medicamentosas podem estar associadas a características do indivíduo, a processos farmacodinâmicos e farmacocinéticos, a propriedades do medicamento, a interações medicamentosas, ou com outras substâncias e, ainda, ao modo de uso (Parand et al., 2016; Freitas, Fonteles, Lima, Bachur \& Carvalho, 2017). O que difere o evento tóxico de uma reação adversa é o fato de que no evento tóxico o dano biológico ocorre, na maioria das vezes, por exposição a doses excessivas de um fármaco (Nobrega et al., 2015). Os fármacos comumente responsáveis pelo processo de intoxicação são: anti-histamínicos, antiparkinsonianos, opióides, anticonvulsivantes, benzodiazepínicos, antidepressivos tricíclicos, betabloqueadores, bloqueadores dos canais de cálcio, salicilatos e antiinflamatórios (Monte, Nunes, Nunes \& Mendes 2016; Zanjani et al., 2016).

Além dos danos individuais que as intoxicações medicamentosas provocam a pessoa intoxicada, os números crescentes deste tipo de intoxicação geram mudanças desfavoráveis ao sistema de saúde uma vez que contribuem para um aumento da demanda dos serviços e consequentemente um aumento nos custos financeiros para o poder público (Rangel \& Francelino, 2018). 
O presente trabalho teve como objetivo analisar o perfil epidemiológico das notificações de intoxicações agudas causadas por medicamentos ocorridas no Piauí, no período de 2007 até 2019.

\section{Metodologia}

Trata-se de um estudo epidemiológico, quantitativo, do tipo descritivo e retrospectivo, onde se adotou os fundamentos metodológicos da epidemiologia que conforme Rouquayrol (1994) é a elaboração do problema epidemiológico, passando por fontes geradoras do mesmo, raciocínio epidemiológico, variáveis e hipóteses epidemiológicas até a arquitetura da investigação. A pesquisa aborda o perfil epidemiológico dos casos de intoxicação exógena por medicamentos no Piauí de 2007 a 2019. A população do estudo foi constituída por todos os casos de intoxicações exógena por medicamentos notificadas pelo serviço de atendimento, diagnosticadas e registradas no SINAN (Sistema de Informações de Agravos de Notificação) no período de 2007 a 2019 no estado do Piauí. Os dados secundários foram coletados das fichas de notificação de intoxicação exóg ena registradas na plataforma do Sistema Nacional de Agravos de Notificação (SINAN), disponibilizado pelo Departamento de Informática do Sistema Único de Saúde (DATASUS) e sendo posteriormente tabulados em planilhas do Microsoft Excel®.

Foram utilizados na pesquisa dados secundários, obtidos no banco de dados do SINAN, os quais são disponibilizados por meio eletrônico no portal do DATASUS do Ministério da Saúde.

A coleta de dados ocorreu entre janeiro e fevereiro de 2021. As variáveis utilizadas nesse estudo foram: ano de notificação, sexo, faixa etária, zona de ocorrência das intoxicações (urbana, rural e periurbana), agente tóxico (medicamento), circunstância, evolução dos casos. Com relação aos indivíduos afetados foram analisados o sexo (masculino e feminino) e idade (<1ano- 80 e mais anos). Foram incluídos no estudo todos os casos notificados no período de 2007 a 2019. Os casos que apresentaram alguma inconsistência ou ausência de alguma informação das variáveis utilizadas na pesquisa e não estavam inclusos no período de estudo foram excluídos.

Para subsidiar a análise dos dados, todas as informações coletadas foram reunidas e posteriormente tabuladas, em planilhas do Microsoft Excel® para realização da análise descritiva e apresentada por meio de tabelas.

No presente estudo foram utilizados dados do tipo secundário, obtidos através do sítio eletrônico do DATASUS na internet, sendo esse de domínio público. Diante disso, torna-se dispensável a apreciação por um Comitê de Ética em Pesquisa (CEP).

Uma das limitações deste estudo decorre do fato de ser retrospectivo e depender da qualidade dos registros, além da presença de muitos casos ignorados que pode prejudicar a melhor análise dos dados.

\section{Resultados}

No Brasil, no período de 2007 a 2019, foram notificados cerca de 509.679 casos de intoxicação exógena por medicamentos de acordo com plataforma DATASUS, sendo que $93.193(18,28 \%)$ desses foram na Região Nordeste e 6.210 (6,66\%) no Estado do Piauí. A Tabela 1 corrobora a frequência dos agentes tóxicos que causam intoxicação exógena e evidencia ainda que os medicamentos foram os principais agentes responsáveis pelo processo de intoxicação correspondendo à $57,16 \%$ das notificações. 
Tabela 1 - Distribuição do número de notificações de intoxicações exógenas no Piauí entre 2007 a 2019 segundo agente tóxico.

\begin{tabular}{lcc}
\hline \multicolumn{1}{c}{ Agente tóxico } & n & $\%$ \\
\hline Medicamentos & 6210 & 57,16 \\
Agrotóxico agrícola & 332 & 3,06 \\
Agrotóxico doméstico & 196 & 1,80 \\
Agrotóxico saúde pública & 29 & 0,27 \\
Raticida & 522 & 4,8 \\
Prod. Veterinário & 193 & 1,50 \\
Prod. uso domiciliar & 727 & 7,34 \\
Cosmético & 220 & 2,03 \\
Prod. Químico & 284 & 2,61 \\
Metal & 3 & 0,03 \\
Drogas de abuso & 507 & 4,67 \\
Planta tóxica & 138 & 1,27 \\
Alimento e bebida & 930 & 8,56 \\
Outro & 533 & 4,91 \\
\hline Total & $\mathbf{1 0 8 6 4 *}$ & $\mathbf{1 0 0}$ \\
\hline *Foram retirados 1.325 casos ignorados (N=12.189). Fonte: \\
DATASUS (2021).
\end{tabular}

A Tabela 2 mostra um aumento progressivo no número de casos de intoxicações medicamentosas registrados no período analisado no estudo variando de 0,32\% em 2007 para 17,74\% em 2019. A menor frequência envolveu o ano de 2007 com $0,32 \%(n=20)$ dos casos enquanto em 2019 houve 1.102 casos $(17,74 \%)$, revelando-se como ano de maior número em valores absolutos. Analisando somente os anos mais recentes, observou-se que entre 2016 ( $n=781$ ) e 2019 (n=1.102) houve um aumento de $5,16 \%$ nos casos.

Tabela 2 - Distribuição dos casos notificados de intoxicações exógenas por medicamentos, no Piauí, segundo ano de ocorrência (período 2007-2019)

\begin{tabular}{ccc}
\hline ANO & $\mathbf{N}$ & $\%$ \\
\hline 2007 & 20 & 0,32 \\
2008 & 61 & 0,98 \\
2009 & 113 & 1,82 \\
2010 & 126 & 2,02 \\
2011 & 182 & 2,93 \\
2012 & 275 & 4,42 \\
2013 & 435 & 7 \\
2014 & 527 & 8,49 \\
2015 & 610 & 9,82 \\
2016 & 781 & 12,58 \\
2017 & 900 & 14,49 \\
2018 & 1080 & 17,39 \\
2019 & 1102 & 17,74 \\
\hline Total & $\mathbf{6 2 1 0}$ & $\mathbf{1 0 0}$ \\
\hline
\end{tabular}

Fonte: DATASUS (2021). 
A analise descritiva que o sexo feminino (71,38\%) na faixa etária de 20-39 (40,43\%) anos é responsável pelo maior número de casos (tabela 3). O estudo descritivo das características demográficas (Tabela 3) revelou que se tratou de eventualidades tipicamente urbanas $(\mathrm{n}=5.189 ; 84,91 \%)$.

Tabela 3 - Distribuição de casos notificados de intoxicações exógenas por medicamentos pelo SUS na plataforma DATASUS, no Piauí, de acordo com gênero, faixa etária e zona.

\begin{tabular}{|c|c|c|}
\hline Sexo & $\mathbf{N}$ & $\%$ \\
\hline Masculino & 1.777 & 28,62 \\
\hline Feminino & 4.433 & 71,38 \\
\hline Total & 6210 & 100 \\
\hline Idade(anos) & $\mathbf{N}$ & $\%$ \\
\hline$<1$ & 148 & 2,38 \\
\hline 01-04 & 732 & 11,79 \\
\hline $05-09$ & 305 & 4,91 \\
\hline $10-14$ & 390 & 6,28 \\
\hline $15-19$ & 1012 & 16,30 \\
\hline $20-39$ & 2510 & 40,43 \\
\hline $40-59$ & 862 & 13,88 \\
\hline $60-64$ & 78 & 1,26 \\
\hline $65-69$ & 58 & 0,93 \\
\hline $70-79$ & 72 & 1,16 \\
\hline $80 \mathrm{e}+$ & 41 & 0,66 \\
\hline Total & $6208 *$ & 100 \\
\hline Zona de residência & $\mathbf{N}$ & $\%$ \\
\hline Urbana & 5189 & 84,91 \\
\hline Rural & 917 & 15,00 \\
\hline Periurbana & 5 & 0,08 \\
\hline Total & 6111* & 100 \\
\hline
\end{tabular}

*Foram excluídos os casos ignorados/branco: zona de residência (n=99), faixa etária $(\mathrm{n}=2)$. Fonte: DATASUS (2021). 
De acordo com a Tabela 4, as principais circunstâncias causais associadas aos casos de intoxicação exógena por medicamentos incluem tentativa de suicídio $(n=3.450)$, seguido do uso terapêutico $(n=721)$, uso acidental ( $n=694)$ e automedicação $(n=343)$.

Tabela 4 - Distribuição da frequência de casos notificados de intoxicação por medicamento no período de 2007 a 2019 segundo circunstância causal no Piauí-PI.

\begin{tabular}{lcc}
\hline \multicolumn{1}{c}{ Circunstâncias } & $\mathbf{n}$ & \% \\
\hline Uso Habitual & 252 & 4,2 \\
Acidental & 694 & 11,57 \\
Ambiental & 2 & 0,03 \\
Uso terapêutico & 721 & 12,02 \\
Prescrição médica & 11 & 0,18 \\
Erro de administração & 128 & 2,13 \\
Automedicação & 343 & 5,72 \\
Abuso & 84 & 1,4 \\
Ingestão de alimento & 15 & 0,25 \\
Tentativa de suicídio & 3450 & 57,52 \\
Tentativa de aborto & 13 & 0,22 \\
Violência/homicídio & 255 & 4,25 \\
Outra & 29 & 0,48 \\
\hline Total & $\mathbf{5 9 9 7 *}$ & $\mathbf{1 0 0}$ \\
\hline *Foram excluídos os casos ignorados/branco: (n=213). Fonte: \\
DATASUS (2021).
\end{tabular}

Quanto à evolução, foram notificados 4.704 casos dos quais 96,75\% (n=4.551) seguiram para cura sem sequela e $1,15 \%(\mathrm{n}=54)$, foram registrados como cura com sequela. Dos outros casos notificados $0,85 \%$ (n=40) evoluíram para óbito em decorrência da intoxicação exógena e $0,08 \%(\mathrm{n}=4)$ para óbito por outra causa. Destaca-se ainda que em $1,17 \%$ dos casos $(n=55)$ houve perda de seguimento da evolução (Tabela 5).

Tabela 5 - Distribuição dos casos no Piauí de acordo com a evolução (N=6.210), no período de 2007 a 2019.

\begin{tabular}{lcc}
\hline \multicolumn{1}{c}{ Evolução } & $\mathbf{n}$ & $\%$ \\
\hline Cura sem sequela & 4551 & 96,75 \\
Cura com sequela & 54 & 1,15 \\
Óbito por intoxicação Exógena & 40 & 0,85 \\
Óbito por outra causa & 4 & 0,08 \\
Perda de Seguimento & 55 & 1,17 \\
\hline Total & $\mathbf{4 7 0 4}$ & $\mathbf{1 0 0}$ \\
\hline
\end{tabular}

*Foram excluídos os casos ignorados/branco na variável evolução $(\mathrm{n}=1.506)$. Fonte: DATASUS (2021).

\section{Discussão}

Os medicamentos foram os principais fatores associados às intoxicações exógenas, apresentando concordância com outros estudos brasileiros (Almeida, 2015; Batista, Sousa, Rocha \& Lacerda, 2015; Monte et al., 2016; Chaves, Viana, Júnior, Silva \& Serra, 2017; Santos \& Boing, 2018; Leite \& Monteiro, 2018). No Piauí o número de casos de intoxicações por medicamento aumentou 17,42\% de 2007 a 2019. Esse crescimento pode ser explicado pela disponibilidade e armazenamento inadequado em domicílio o que pode favorecer a automedicação. Esse número também pode ser explicado por outros fatores de grande relevância como os erros de automedicações, uso abusivo, acidente individual, tentativas de suicídio e uso 
terapêutico, que podem levar ao aumento significativo do número de casos de intoxicação e letalidade por causas evitáveis (Santos \& Boing, 2018; Leite \& Monteiro, 2018).

Com relação ao sexo, o público feminino apresentou um maior número de notificações justificado pelo fato que as mulheres realizam mais práticas de automedicação corroborando com muitas pesquisas realizadas no país (Almeida, 2015; Batista et al., 2015; Monte et al., 2016; Chaves et al., 2017; Leite \& Monteiro, 2018). Além disso, o público feminino também pode adquirir intoxicações por buscarem de forma mais frequente o atendimento de saúde em comparação aos homens (Cardos, Amorim, Robles \& Rangel, 2018). Ademais mulheres podem utilizar os medicamentos de forma abusiva, por adquirilos em grandes quantidades para armazenamento em casa, prática conhecida como "farmácia caseira" (Domingues et al., 2015; Freitas et al., 2017). Por essas razões pode-se explicar a maior frequência de mulheres nos casos de intoxicação.

Com relação à zona de residência, a zona urbana foi a mais destacada e um dos principais fatores que levam a ocorrência desses casos está relacionado à facilidade de ter acesso aos medicamentos pelo grande número de farmácias nas cidades. Outros fatores contribuintes são a frágil regulação da publicidade acerca do medicamento, a facilidade na aquisição de fármacos sem prescrição médica, a deficiência de legislação específica sobre embalagens seguras, escassas iniciativas de desenvolvimento da atenção farmacêutica, o padrão do consumo de medicamentos pela população (automedicação, polifarmácia, uso indevido e indiscriminado, principalmente de psicotrópicos e antibióticos), e o aumento do número de doenças crônicas na população aumentando o consumo de medicamentos (Freitas et al.,2017; Gonçalves et al.; 2017; Monte et al., 2016).

O predomínio de adultos jovens nesta pesquisa se deve possivelmente, ao fato de que essa é a idade mais produtiva para o ser humano, onde o dia a dia leva a utilização de fármacos de maneira errônea, principalmente, por automedicação. Os grupos etários de 15-19 anos (16,30\%) e de 20-39 anos (40,43\%) tem sido abordados em diversas literaturas como os grupos mais suscetíveis as tentativas de suicídio por uma superdosagem de medicamentos (Almeida, 2015; Batista et al., 2015; Monte et al.,2016; Chaves et al., 2017). Dessa forma o que se pode entender é que a maioria dos jovens praticam a automedicação utilizando os medicamentos de forma errônea. Somado a isso, o aumento das doenças crônicas na população leva a intoxicações devido à grande quantidade de fármacos utilizados principalmente pelo público 40-59 anos o qual ocupa o terceiro lugar entre as faixas etárias que apresentam intoxicações por medicamento (Domingues et al., 2015; Gonçalves et al.;2017; Batista et al., 2017; Santos \& Boing, 2018).

Vale destacar que público pediátrico ( $<1$ ano; 1-5anos; 5-9 anos) representou 19,08\% ( $\mathrm{n}=1.185)$ do total de 6.208 casos notificados. Para esse grupo, alguns estudos descrevem que o pico de ocorrência na faixa de 1 a 4 anos se deve a etapa do desenvolvimento físico e cognitivo da criança. Dessa forma, a criança começa a se locomover sozinha pelo ambiente e por questão de curiosidade pode ingerir medicamentos presentes em casa. Assim, devido ao hábito de levar objetos a boca, há maiores riscos do desenvolvimento de um quadro de intoxicação, já que crianças desconhecem os riscos e fatalidades do uso desses fármacos. À medida que ocorre o maior desenvolvimento cognitivo da criança, a frequência de casos de intoxicações diminui (Domingos et al., 2016; Batista et al., 2017; Lopes et al., 2019).

Em relação à circunstância do uso de medicamento, registrou-se 57,52\% envolvendo tentativa de suicídio predominantemente entre o sexo feminino, destacando esse público como bastante vulnerável. Alguns estudos relatam que os fatores determinantes são a falta de conhecimento das propriedades dos medicamentos e o perfil psicológico das vítimas, sendo esse o principal fator relacionado ao suicídio (Rangel \& Francelino, 2018; Gonçalves et al., 2017; Monte et al., 2016). Outras circunstâncias responsáveis por um número expressivo das notificações foram relacionadas aos usos terapêutico e acidental. Esses dois fatores são relacionados ao maior número de doenças crônicas na população levando ao uso contínuo de medicamentos e a prática de farmácia "caseira"; o uso acidental pode ser justificado em grande parte por conta do público 
pediátrico que ingere os medicamentos de forma não intencional (Maior et al., 2017; Lopes et al., 2019; Mendonça et al., 2016).

O presente estudo descreve que a maioria dos casos notificados evoluiu para cura sem sequela (Monte et al., 2016), os casos ignorados foram retirados do valor total, mas há de se ressalvar que eles representam um valor elevado. Essa informação representa uma deficiência do serviço em notificar adequadamente as ocorrências (Rangel \& Francelino, 2018).

O número de notificações de intoxicações exógenas por medicamentos atualmente envolve desde a funcionalidade do sistema de saúde até a facilidade no acesso aos medicamentos pela população. Além disso, os profissionais da saúde são os principais mediadores envolvidos na prevenção devido ao contato mais frequente e direto com a população. $\mathrm{O}$ uso indevido de fármacos é um problema de saúde pública que afeta não só a sociedade brasileira, mas também inúmeros países (Batista et al., 2015; Monte et al.,2016; Santos \& Boing,2018; Leite e Monteiro, 2018).

\section{Conclusão}

As intoxicações exógenas por medicamentos apresentam um número expressivo no estado do Piauí, merecendo uma atenção especial pelos profissionais de saúde pública. O público feminino apresentou um maior número de notificações justificado pelo fato que as mulheres realizam mais práticas de automedicação, com relação à faixa etária os adultos jovens predominaram e a zona urbana foi a mais destacada. As estatísticas do perfil de intoxicações por medicamentos são importantes fontes de dados para conhecer a problemática das intoxicações medicamentosas, analisar tendências, indicar prioridades e avaliar programas. Nesse sentido, o presente estudo visa contribuir para o aprimoramento das políticas públicas de saúde com foco na prevenção, orientação e adoção de medidas que minimizem este quadro, com atenção especial para público adulto-jovem e feminino que são os principais responsáveis pelas notificações de intoxicação medicamentosa no Piauí.

Restringir o acesso das crianças aos medicamentos e a adoção de embalagens especiais é muito importante, por meio do fomento à cultura de segurança doméstica. Se medidas educacionais são importantes para o aumento da segurança doméstica, medidas regulatórias sobre o uso racional são essenciais para a diminuição das intoxicações medicamentosas.

Destaca-se ainda o papel da equipe da atenção básica, pois as equipes das unidades básicas de saúde possuem um maior contato com a comunidade. Dessa forma, podem fornecer orientações sobre os medicamentos, seus efeitos adversos, além de orientar com relação ao cuidado para que as crianças não consumam medicamentos de forma acidental, a necessidade de intervenções educativas com a finalidade de informar sobre os riscos do uso indevido de qualquer medicação. Além disso, deve-se realizar ações de prevenção ao suicídio devido ser esta a principal circunstância que ocasiona intoxicação exógena por medicamentos.

\section{Referências}

Almeida, G. F. Perfil Epidemiológico das intoxicações por medicamentos em Campina Grande (2015) 35f. Trabalho de conclusão de curso-Universidade Estadual da .

Batista, L. A., Sousa, M. D. R., Rocha, R. J. \& Lacerda, E. M. C. B. (2017) Epidemiological profile of intoxication cases reported in the State of Maranhão. Revista de investigação biomédica, 9(2):129-137.

Cardos, D. V., Amorim, D. S., Robles, A. J. M. \& Rangel, T. L. V. (2018) A invisibilidade dos homens nas unidades de atenção primária a saúde no brasil de acordo com estudos realizados nos últimos dez anos. Jornada de iniciação cientifíca da UNIFACIG, 4.

Chaves, L. H. S., Viana, A. C., Júnior, W. P. M., Silva, A. L. \& Serra, L. C (2017). Exogenous intoxication by medications: epidemiological aspects of notified cases between 2011 and 2015 in Maranhão, ReonFacema, 3 (2) :477-482.

Domingues, P. H. F., Galvão, T. F., Andrade, K. R. C., Sá, P. T. T., Silva, M. T. \& Pereira, M. G. (2015). Prevalência da automedicação na população adulta do Brasil: revisão sistemática. Revista Saúde Pública,49(36)

Domingos, S. A., Borghesan, N. B. A., Merino, M. F. G. L. \& Higarashi,I. H.(2016). Poison-related hospitalizations of children aged 0-14 at a teaching hospital in Southern Brazil, 2006-2011. Revista Epidemiologia e Serviços de Saúde, 25(2) :343-350. 
Freitas, J. A. B., Fonteles, M. M. F., Lima, M. E. S., Bachur, T. P. R. \& Carvalho, T. M. J. P. (2017) Over-the-counter drugs: consumption profile and toxic risks of acetaminophen. Revinter, 10 (3): 134-154.

Geulayov, G., Ferrey, A., Casey, D., Wells, C., Fuller, A., Bankhead, C., Gunnell, D., Clements, C., Kapur, N., Ness, J., Waters, K. \& Hawton, K. (2018) Relative toxicity of benzodiazepines and hypnotics commonly used for self-poisoning: An epidemiological study of fatal toxicity and case fatality.Journal of Psychopharmacology, 32 (6): 654-662.

Gonçalves, C. A, Santos, V. A., Gonçalves, C. A., Sarturi, L \& Junior, A. T. T. (2017). Intoxicação medicamentosa: relacionada ao uso indiscriminado de medicamentos. Revista Científica da Faculdade de Educação e Meio Ambiente, 8 (1):.135-143.

Huynh, A.,Cairns, R, Brown, J., Lynch, A. M. Robinson, J., Wylie, C., Buckley, N. A. \& Dawson, A. H. (2018). Patterns of poisoning exposure at diferente ages: the 2015 annual report of the Australian Poisons Information Centres. The Medical Journal of Astraulia, 209 (2).

Leite, M. M. S, Monteiro, A. B. (2018) Análise das intoxicações medicamentosas no estado da paraíba-brasil em 2017. Journal of Biology \& Pharmacy and Agricultural Management, 14(2).

Lopes, D. S., Bispo, D. C., Carvalho, F. S., Macêdo, K. P. C., Freitas, R. L., Oliveira, E. H. \& Sousa, J. A. (2019) Intoxicações por medicamentos em pacientes pediatricos: análise na cidade de Teresina, Piauí. Brazilian Journal of Surgery and Clinical Research - BJSCR, 27 (1):33-36.

Maior, M. C. L. S., Castro, C. G. S. O \& Andrade, C. L. T. (2017) Internações por intoxicações medicamentosas em crianças menores de cinco anos no Brasil, 2003-2012*. Revista de Epidemiologia e Serviços de Saúde, 26(4):771-782.

Mendonça, D. R., Menezes, M. S., Matos, M. A. A., Rebouças, D. S., Filho, J. N. C., Assis, R. S. \& Carneiro, L. (2016) Acute Poisoning in Children in Bahia, Brazil. Global Pediatric Health, 3:1-7.

Monte, B. S.,Nunes, M. S. T.,Nunes, M. D. S. \& Mendes, C. M. M. (2016) Epidemiological study of intoxications by medications registered by the toxicology information center of Piauí - citox: 2007 to 2012. Revista Interdisciplinar, 9 (3): 96-104.

Moyo, A. P., Zhao, X. \& Thorpe, C. T. (2019). Patterns of opioid prescriptions received prior to unintentional prescription opioid overdose death among Veterans. Research in Social and Administrative Pharmacy, $15: 1007-1013$

Ministério da saúde. Secretaria de Vigilância em Saúde. Departamento de Vigilância Epidemiológica. Sistema de Informação de Agravos de Notificação Sinan, 2017

Ministério da saúde. Portaria GM/MS No 104, de 25 de janeiro de 2011. Define, a relação de doenças, agravos e eventos em saúde pública de notificação compulsória em todo o território nacional e estabelece fluxo, critérios, responsabilidades e atribuições aos profissionais e serviços de saúde. Diário Oficial da União, Brasília-DF, 26 de Janeiro de 2011, p.37.

Nguyen, M. B., Pizon, A. F., Branas, C. C. \& Anthony, F. (2016). Regional variations in pediatric medication exposure: Spatial analysis of poison center utilization in western Pennsylvania. Clinical Toxicology, 54(1):47-52.

Nóbrega, H. O. S., Costa, A. M. P. \& Mariz, S. R. (2015). Intoxicações por medicamentos: uma revisão sistemática com abordagem nas síndromes tóxicas. Revista saúde e ciência on line, 4(2):109-119.

Parand, A., Garfield, S. \& Vincent, C. Carers' Medication Administration Errors in the Domiciliary Setting: A Systematic Review. PLOS ONE, 11(12).

Peixoto, N. H.\& Ferreira, L. S. (2013) Higiene ocupacional III - Santa Maria: Universidade Federal de Santa Maria. Colégio Técnico Industrial de Santa Maria. Rede e-Tec.

Rangel, N. L. \& Francelino, E. V. (2018.) Caracterização do Perfil das Intoxicações Medicamentosas no Brasil, durante 2013 a 2016. Id on Line Revista Multidisciplinar e de Psicologia., 12(42):121-135.

Rouquayrol, M. Z., Goldbaum, M. (1994) Epidemiologia, história natural e prevenção de doenças. Epidemiologia e saúde, 17-35.

Santos, G. A. S. \& Boing, A. C. (2019). Drug Intoxication In Brazil: An Analysis Of The Cases Registered From 2007 To 2017, Paripex - Indian Journal of Research, 8.

Santos, R. R., Neto, O. P. A. \& Cunha, C. M. (2015). Perfil de vítimas de intoxicações exógenas agudas e assistência de enfermagem. Revista Enfermagem Atenção Saúde. 4(2):45-55.

Sinitox - Sistema Nacional de Informação Tóxico-Farmacológica. Centro de Informação Científica e Tecnológica, Fundação Oswaldo Cruz. Casos, Óbitos e Letalidade de Intoxicação Humana por Agente e por Região. Brasil, 2017. < https://sinitox.icict.fiocruz.br/sites/sinitox.icict.fiocruz.br/files//Brasil3.pdf>.

Toscano, M. M., Landim, J. T. A., Rocha, A. B. \& Muñoz, R. L. S. (2016). Intoxicações exógenas agudas registradas em centro de assistência toxicológica. Revista Saúde e Pesquisa, 9(3):425-432.

Who. International Programme on Chemical Safety, II. Technical guidance - 4. Analytical toxicology and other laboratory services. http://www.who.int/ipcs/publications/training_poisons/guidelines_poison_control/en/index4.html.

Zanjani, F.; Smith, R.; Slavova, S., Charnigo, R., Schoenberg, N., Martin, C. \& Clayton,R. (2016) Concurrent alcohol and medication poisoning hospital admissions among older rural and urban residents. The American Journal of Drug and Alcohol Abuse, 42(4):422-30. 